\title{
Indicadores de sostenibilidad para la industria minera extractiva en Uige, Angola
}

\section{Sustainability indicators for the extractive mining industry in Uige, Angola}

\author{
${ }^{1}$ Instituto Superior de Ciencas de Educación de Uige, Angola. \\ ${ }^{2}$ Universidad de Moa, Holguín, Cuba. \\ *Autor para la correspondencia: andrebambi2@gmail.com
}

André Afonso-Bambi ${ }^{1 *}$, Juan Manuel Montero-Peña ${ }^{2}$, Roberto Watson-Quesada ${ }^{2}$

\section{Resumen}

En la provincia de Uige (Angola) no existe un procedimiento que permita evaluar la sostenibilidad de la industria minera extractiva de materiales para la construcción. Esta investigación tuvo el propósito de proponer un sistema de indicadores de sostenibilidad que sirva de instrumento para medir el grado de desarrollo sostenible en el sector minero, teniendo en cuenta el estado técnico y organizativo de las canteras y las dimensiones ambiental, económica y social. Mediante el empleo de los métodos empíricos de encuesta y entrevista se llevó a cabo el diagnóstico en los escenarios y comunidades mineras de Uige y se crearon cuatro tipos de indicadores: 1) de gestión minera, 2) de crecimiento económico, 3) de compensación y 4) de desarrollo, a partir de los cuales se calculó el índice de sostenibilidad global, cuyo valor reveló la no sostenibilidad de la empresa minera en la provincia. A partir de esta información se proponen acciones para cada uno de los actores que conforman el escenario socioeconómico en Uige.

Palabras clave: industria minera; indicadores de sostenibilidad; canteras; materiales para la construcción; desarrollo sostenible.

\section{Abstract}

There is no procedure for evaluating the sustainability of the extractive mining industry of construction materials in the province of Uige (Angola). The purpose of this research is to propose a system of sustainability 
indicators that work as an instrument measuring the sustainable development degree in the mining sector, taking into account the technical and organizational status of the quarries and the environmental, economic and social dimensions. The diagnosis in Uige mining scenarios and communities was carried out by using empirical methods such as: survey and interview and four types of indicators were created: 1) mining management, 2) economic growth, 3) compensation and 4) development, such value revealed the non-sustainability of the mining company in the province. For each driver that makes up the socioeconomic scenario in Uige are proposed actions based on this information..

Keywords: mining industry; sustainability indicators; quarries; construction materials; sustainable development.

\section{INTRODUCCIÓN}

La aparición del concepto desarrollo sostenible es el resultado de una emergencia planetaria ante la explotación indiscriminada de los recursos del planeta Tierra por parte de sus habitantes. Surgido como elaboración teórica en una etapa de auge del desarrollo de la ciencia y la tecnología, y de visualización de sus impactos, propone un tipo de pacto intergeneracional que busca conciliar las contradicciones entre el crecimiento desenfrenado de la producción, los hábitos de consumo insostenibles -incluso dentro de los esquemas de producción más eficientes desde el punto de vista tecnológico- y los evidentes límites de la naturaleza para ofrecer recursos naturales de manera sostenida.

La satisfacción de las necesidades de las presentes y futuras generaciones sugiere el establecimiento de medidas así como su evaluación en cada comunidad en una dimensión espacio-temporal.

Por otra parte, las capacidades del medio ambiente para satisfacer las necesidades de las presentes y futuras generaciones están mediadas por muchos factores, entre ellos la demanda inconmensurable de materiales para la construcción. En Angola esta situación está signada por la necesidad de acometer numerosas obras para la recuperación de toda la infraestructura económica y social afectada por la guerra civil.

En cuanto a la sostenibilidad de la industria minera se plantea lo siguiente:

"la importancia de crear un enfoque responsable y sostenible del desarrollo de la minería nunca ha sido más clara (...) aunque el papel de la minería en el desarrollo económico cada vez cuenta con más 
reconocimiento, se sigue debatiendo sobre cómo debería ser la minería" (Wong y Tost 2013, s. p.).

En países como Estados Unidos, Canadá, Perú y Chile, con una estructura normativa bien definida, la temática de los indicadores es abordada desde una mirada sistémica, aunque actualmente las dinámicas globales obedecen al modelo capitalista de producción a gran escala, acumulación, derroche y despojo (La Rotta y Torres 2017).

En la literatura es posible encontrar numerosos sistemas de indicadores de sostenibilidad en la minería. Para Carmona-García, Cardona-Trujillo y Restrepo-Tarquino (2017) en la sostenibilidad minera los esfuerzos están orientados hacia el enfoque de la sostenibilidad blanda, tomando como base la integración de las tres dimensiones del desarrollo: económico, ambiental y social. Sin embargo, la operacionalización de estos indicadores carece aún de una dinámica positiva que sirva como referencia obligada para los proyectos mineros.

En Cuba, los indicadores de sostenibilidad en la minería se han trabajado sobre todo en la gestión ambiental de la extracción de los materiales para la construcción, como exponen Reynaldo-Arguelles (2012), Millán (2013), Montes de Oca y Ulloa (2013) y Araña (2015). En Colombia el tema ha sido abordado, entre otros, por Arango y Olaya (2012), Saade (2013) y Urán (2013); mientras que en Chile por Sturla y Muñoz (2014).

Otro aspecto importante a tener en cuenta al abordar los indicadores de sostenibilidad en la minería es la elaboración de planes para el cierre de minas; sobre esto han investigado autores como Parravicini (2015), Montero-Matos, Restrepo-Baena y Otaño-Nogel (2017), entre otros.

Sin embargo, en Angola se ha hecho y escrito muy poco, siendo un tema novedoso en el que mayormente trabajan doctorantes y maestrantes en universidades extranjeras.

Concepciones sobre indicadores de sostenibilidad existen varias. Para Zúñiga, Benavides y Arnáez (2015) un indicador de sostenibilidad es un instrumento que se diseña para medir si los cambios que se están poniendo en práctica realmente contribuyen con el desarrollo sostenible.

Para Montero-Peña (2009) un indicador de sostenibilidad es la estadística o parámetro que proporciona información y tendencias de las condiciones de desarrollo de las diferentes actividades económicas y su influencia en el medio ambiente y en el desarrollo de acciones para el mantenimiento de las condiciones ambientales, sociales y tecnológicas. 
En la provincia angolana de Uige, la industria extractiva minera de explotación de materiales para la construcción ha contribuido mucho al plan de reconstrucción y recuperación de infraestructuras públicas y privadas en la provincia, sin embargo, el índice de sostenibilidad en el sector minero es relativamente bajo conforme a los resultados del test aplicado a las empresas diagnosticadas en esta investigación.

Se ha podido determinar que no se conocen realmente varios aspectos geológicos y geotécnicos que influyen en los parámetros mineros, técnicos y organizativos de las canteras para la realización de una explotación racional y eficiente.

En este sentido, Herbert (2006) afirma que las características geológicas, geotécnicas e hidrogeológicas del yacimiento establecen el diseño, la selección del método de explotación, el dimensionamiento geométrico de la mina, la determinación del ritmo anual de producción, la ley de corte y la secuencia de extracción.

En el escenario minero de Uige el cumplimiento de la gestión minera no obedece realmente a las dimensiones del desarrollo sostenible, al carecer de proyectos de rehabilitación mineroambiental dirigidos a compensar a la sociedad por los impactos que esta actividad minera ocasiona.

El control de producción de las empresas no es eficaz y la relación empresacomunidad no se ha convertido en una palanca del desarrollo comunitario. En la presente investigación se ha podido verificar que las empresas violan varios lineamientos del código minero angolano.

Por otra parte, las instituciones académicas no participan en la resolución de los problemas de las comunidades. La alianza existente entre gobiernoempresa-comunidad es disfuncional, ello constituye una amenaza para el logro de la sostenibilidad.

Ante esta problemática en la provincia de Uige urge una propuesta de indicadores que contribuya realmente al desarrollo sostenible de las comunidades que viven en la zona donde tiene lugar la industria extractiva de minerales para la construcción. El objetivo de este estudio es precisamente proponer indicadores para evaluar la sostenibilidad de esta industria en la provincia angolana de Uige.

\section{METODOLOGÍA}

Para la localización de los espacios mineros, incluyendo las canteras en estado de abandono, se utilizó GPS map 62s. La Tabla 1 describe el estado 
de las canteras georreferenciadas. Para la ubicación de los escenarios mineros en el mapa (Figura 1) se utilizó el software QGIS3.0.2.

Tabla 1. Georreferenciamiento de los espacios mineros industriales

\begin{tabular}{|c|c|c|c|c|}
\hline $\mathbf{N}^{\circ}$ & Material & Localidad & $\begin{array}{c}\text { Coordenadas } \\
\text { Long./Lat. }\end{array}$ & Estado \\
\hline 01 & Caliza & Quimassabi & $15^{\circ} 02^{\prime} 34^{\prime \prime} 7^{\circ} 52^{\prime} 40^{\prime \prime}$ & En operación \\
\hline 02 & Caliza & Pedreira & $15^{\circ} 04^{\prime} 49^{\prime \prime} 7^{\circ} 37^{\prime} 47^{\prime \prime}$ & Abandonada \\
\hline 03 & Caliza & Pedreira & $15^{\circ} 04^{\prime} 53^{\prime \prime} 7^{\circ} 37^{\prime} 53^{\prime \prime}$ & Abandonada \\
\hline 04 & Caliza & Katuhula & $15^{\circ} 05^{\prime} 07^{\prime \prime} 7 \circ 33^{\prime} 60^{\prime \prime}$ & Abandonada \\
\hline 05 & Caliza & Kissanga & $14^{\circ} 59^{\prime} 28^{\prime \prime} 7^{\circ} 43^{\prime} 31^{\prime \prime}$ & Abandonada \\
\hline 06 & Caliza & Makaly & $14^{\circ} 46^{\prime} 31^{\prime \prime} 7^{\circ} 12^{\prime} 34^{\prime \prime}$ & Abandonada \\
\hline 07 & Laterite & Kimbengui & $14^{\circ} 46^{\prime} 18^{\prime \prime} 7^{\circ} 14^{\prime} 49^{\prime \prime}$ & En operación \\
\hline 08 & Argila & Banza Negage & $15^{\circ} 16^{\prime} 14^{\prime \prime} 7 \circ 47^{\prime} 10^{\prime \prime}$ & En operación \\
\hline 09 & Caliza & Bengo & $15^{\circ} 15^{\prime} 56^{\prime \prime} 7 \circ 48^{\prime} 42^{\prime \prime}$ & Abandonada \\
\hline 10 & Caliza & Nganakamana & $15^{\circ} 06^{\prime} 08^{\prime \prime} 7 \circ 35^{\prime} 15^{\prime \prime}$ & En operación \\
\hline 11 & Caliza & Mbabi & $15^{\circ} 04^{\prime} 33^{\prime \prime} 77^{\circ} 32^{\prime} 49^{\prime \prime}$ & Abandonada \\
\hline 12 & Caliza & Tomessa & $15^{\circ} 04^{\prime} 44^{\prime \prime} 7^{\circ} 36^{\prime} 07^{\prime \prime}$ & En operación \\
\hline 13 & Caliza & Banzo polo & $15^{\circ} 05^{\prime} 26^{\prime \prime} 77^{\circ} 36^{\prime} 58^{\prime \prime}$ & En operación \\
\hline 14 & Caliza & Henda & $15^{\circ} 06^{\prime} 40^{\prime \prime} 77^{\circ} 37^{\prime} 44^{\prime \prime}$ & En operación \\
\hline
\end{tabular}

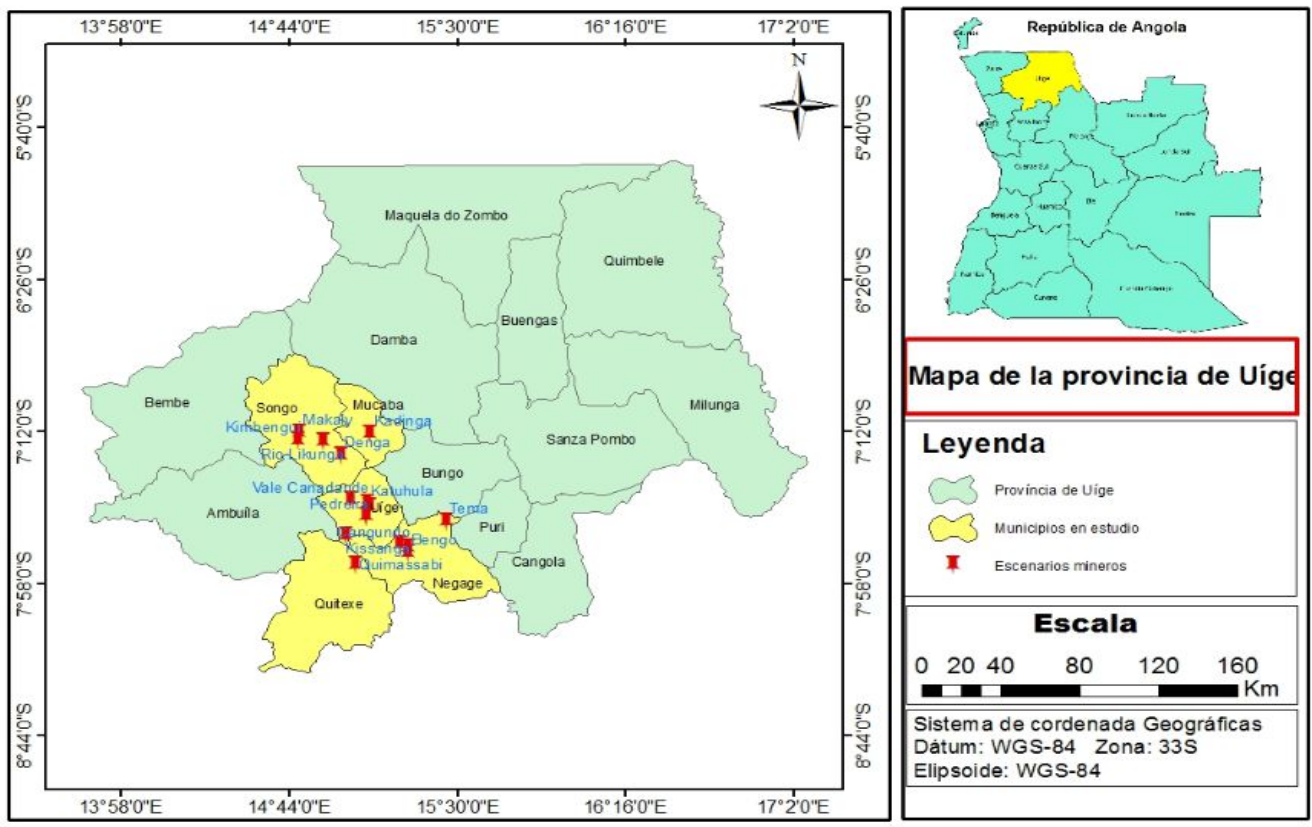

Figura 1. Escenarios mineros estudiados. 
Para proponer los indicadores de sostenibilidad en la minería de materiales para la construcción se revisaron varios sistemas de indicadores resultantes de trabajos realizados por investigadores de esta temática en distintas organizaciones. Se aplicó, además, un cuestionario a tres comunidades a fin de verificar los problemas predominantes en estas comunidades que viven en las zonas de desarrollo de la minería.

Para determinar el índice de sostenibilidad de las empresas se aplicó un diagnóstico a dos empresas locales, usando la metodología de Carvajal y González (2003), la cual establece que si el índice de sostenibilidad global (ISG) ISG $>50 \%$ la actividad extractiva se encontraría en el campo de la sostenibilidad y sería tanto más sustentable cuanto más se aproxime a $100 \%$. En cambio, si el ISG $<50 \%$ la actividad extractiva entraría en el campo de la no sostenibilidad y, por tanto, la empresa minera debería de revisar a fondo las acciones que lleva a cabo. Se calculó el índice de sostenibilidad global como la sumatoria del valor de cada uno de los indicadores dividido entre la suma total de acciones de los indicadores.

La propuesta de indicadores se aplicó a una empresa activa existente en la provincia para medir el índice de sostenibilidad de actividad minera mediante un cuestionario específico para cada indicador (Tablas 2, 3, 4 y 5). Se consideró valor uno (1) cuando el indicador se cumple y cero (0) cuando no.

\section{RESULTADOS Y DISCUSIÓN}

A partir de los resultados del diagnóstico se propusieron cuatro indicadores de sostenibilidad:

1. Indicador geológico minero.

2. Indicador de crecimiento económico.

3. Indicador de compensación.

4. Indicador de desarrollo.

Los índices de sostenibilidad pueden ser evaluados de acuerdo con la fórmula propuesta por Carvajal y González (2003). Esta metodología se basa en la realización de un test de sostenibilidad a la empresa minera objeto de estudio. El aporte del test son los indicadores de sostenibilidad, cada uno de los cuales se evalúa con respuestas sí/no a una serie de preguntas sencillas pero que responden a acciones claves por parte de la empresa minera. 


\subsection{Resultado a partir del diagnóstico de una de las empresas en Uige}

Las Tablas 2, 3, 4 y 5 exponen el número de acciones que componen cada indicador y los resultados del cuestionario aplicado en una de las empresas que opera en la provincia.

Tabla 2. Geológico minero

\begin{tabular}{|c|c|c|}
\hline \multirow{2}{*}{ Cuestionario } & \multicolumn{2}{|c|}{ Cumple } \\
\hline & Sí & No \\
\hline ¿Se conocen las características tectónicas del yacimiento? & & $\mathrm{X}$ \\
\hline ¿Se conocen las características hidrogeológicas del yacimiento? & & $\mathrm{X}$ \\
\hline ¿Se conocen las características geotécnicas del yacimiento? & & $\mathrm{X}$ \\
\hline ¿Se conocen las condiciones para la estimación de la reserva? & & $\mathrm{X}$ \\
\hline $\begin{array}{l}\text { ¿Se ha determinado la capacidad anual de producción y vida útil } \\
\text { de la cantera? }\end{array}$ & $\mathrm{X}$ & \\
\hline ¿Se conoce la cantidad de empleados existente en la cantera? & $\mathrm{X}$ & \\
\hline ¿Se conoce el rendimiento del personal en la cantera? & $\mathrm{X}$ & \\
\hline ¿Se ha construido escombrera en la cantera? & & $\mathrm{x}$ \\
\hline ¿Se ha elaborado un plan calendario en la cantera? & & $\mathrm{x}$ \\
\hline $\begin{array}{l}\text { ¿Se ha calculado el equipamiento minero y de transporte de } \\
\text { acuerdo con los volúmenes de mineral a explotar? }\end{array}$ & & $\mathrm{x}$ \\
\hline $\begin{array}{c}\text { ¿Los elementos del sistema de explotación obedecen los factores } \\
\text { a tener en cuenta en su proyección? }\end{array}$ & & $\mathrm{X}$ \\
\hline$\Sigma$ & 3 & 8 \\
\hline
\end{tabular}

\subsection{Indicador geológico minero (IGM)}

Este indicador expone de forma breve y concisa las principales características geológicas del yacimiento, constituyendo un marco referencial para la proyección de la explotación de este. El indicador ofrece asimismo varios criterios que permiten la cuantificación o cualificación de las condiciones geológicas y parámetros de las labores mineras que influyen en la eficiencia y eficacia de la explotación minera.

\section{Criterios del indicador}

- TY- La tectónica del yacimiento describe los elementos tectónicos más importantes que pueden incidir en la explotación correcta del yacimiento. 
- HY- La hidrogeología del yacimiento ofrece una caracterización, que incluye la posición de los horizontes acuíferos, cálculo de las afluencias de agua, así como otros datos que permitan definir en el proyecto las principales soluciones de drenaje durante la explotación.

- CGM- Las características geotécnicas del macizo exponen las principales propiedades físico-mecánicas y el agrietamiento del mineral y de las rocas encajantes, calidad de la roca y del macizo.

- CER- Las condiciones para la estimación de los recursos ofrecen los parámetros por los cuales fueron estimados los recursos minerales.

- CVU- La capacidad anual de producción y vida útil del yacimiento ofrece la capacidad anual de la mina de acuerdo con la capacidad anual de procesamiento de la planta de beneficio.

- CEM- La cantidad de empleados en la mina abarca todo el personal.

- RPM- El rendimiento del personal en la mina establece la relación entre la capacidad de producción de la mina y el personal empleado.

- CE- La construcción de escombrera ofrece los principales parámetros de construcción de esta, así como la decisión de los lugares de ubicación del escombro durante todo el tiempo de explotación del yacimiento.

- PCM- El plan calendario de la minería expone el plan de extracción y transportación de mineral y escombro por año, diario y por turnos, así como una explicación detallada de cada año.

- EMT- Los cálculos del equipamiento minero y de transporte ofrece la fundamentación para la selección y adquisición de los equipos, así como la relación de los mismos para las distintas actividades de la minería.

- ESE- Los elementos del sistema de explotación establece el cumplimiento del dimensionamiento idóneo de los principales elementos del sistema de explotación.

El índice de sostenibilidad geológico minero (ISGM, en porcentaje) se calcula como: 


$$
\begin{aligned}
& I S G M(\%)=\sum \frac{[(T Y+H Y+C G M+C E R+C V U+C E M+R P M+C E+P C M+E M T+E S E)]}{11} X 100 \\
& I S G M(\%)=\sum \frac{[(0+0+0+0+1+1+1+0+0+0+0)]}{11} X 100 \\
& I S G M=27,27 \%
\end{aligned}
$$

Tabla 3. Crecimiento económico

\begin{tabular}{cl}
\hline Cuestionario & \multicolumn{1}{c}{ Cumple } \\
\cline { 2 - 2 } $\begin{array}{c}\text { ¿Se conoce el monto de inversión realizado para la puesta en marcha } \\
\text { del proyecto? }\end{array}$ & $\mathrm{No}$ \\
\hline ¿Se conoce el costo de operación por actividad? & $\mathrm{X}$ \\
¿Se conoce el costo de producción de la materia prima? & $\mathrm{X}$ \\
¿La tasa de ingreso anual es satisfactoria? & $\mathrm{X}$ \\
¿La rentabilidad anual es satisfactoria? & $\mathrm{X}$ \\
¿El aporte fiscal de la empresa es aceptable? & $\mathrm{X}$ \\
¿La superficie territorial cedida al proyecto es satisfactoria? & $\mathrm{X}$ \\
¿La tendencia en la variación de la producción anual es positiva? & $\mathrm{X}$ \\
¿La tendencia en la variación de los precios anual es favorable? & $\mathrm{X}$ \\
¿La tendencia en la demanda anual es favorable? & $\mathrm{X}$ \\
$\Sigma$ & 9
\end{tabular}

\subsection{Indicador de crecimiento económico (CE)}

Los indicadores de crecimiento en este caso ofrecen los costos en toneladas o en metros cúbicos realizados durante las distintas actividades de la mina, incluyendo la fuerza de trabajo, mantenimiento, combustibles, la ganancia de la empresa, entre otros. Este indicador debe medir, incluso, el aporte económico de la empresa al PIB y a la comunidad en la cual se encuentra enclavado el yacimiento.

\section{Criterios del indicador}

- MI- El monto de inversión ofrece los capitales de las inversiones realizadas en la ejecución del proyecto minero por actividad.

- CO- El costo de operación por actividad establece los gastos realizados en cada actividad. 
- CP- El costo de la producción establece la relación entre el costo total de la empresa y el total de mineral producido.

- TI- La tasa de ingreso anual establece la cantidad de capital obtenido por ventas durante un año.

- RE- La rentabilidad de la empresa establece la diferencia entre los ingresos obtenidos y el costo de producción del mineral.

- AF- El aporte fiscal de la empresa establece el cumplimiento de todos los compromisos fiscales de la empresa hacia el estado para el incremento de PIB.

- ST- La superficie territorial cedida al proyecto establece el registro de la intervención territorial de la actividad minera en la región.

- VP- La variación de la producción establece la relación entre el aumento o disminución de la producción en periodo determinado comparativamente al periodo anterior.

- VPr- La variación de los precios establece la relación entre el aumento o disminución de los precios en periodo determinado comparativamente al periodo anterior.

- $\mathbf{T M}$ - La tendencia del mercado establece la cantidad de toneladas en metros cúbicos vendidos en un periodo determinado de tiempo comparativamente al periodo anterior.

El índice de sostenibilidad de crecimiento económico (ISCE, en porcentaje) se calcula como:

$$
\begin{aligned}
& \operatorname{ISCE}(\%)=\sum \frac{[(M I++C O+C P+T I+R+A F+S T+V P+V P r+T M)}{10} X 100 \\
& \operatorname{ISCE}()=\sum \frac{[(1+0+1+1+1+1+1+1+1+1)]}{10} \times 100 \\
& \operatorname{ISCE}=90 \%
\end{aligned}
$$


Tabla 4. Compensación

\begin{tabular}{|c|c|c|}
\hline \multirow{2}{*}{ Cuestionario } & \multicolumn{2}{|c|}{ Cumple } \\
\hline & Sí & No \\
\hline ¿Se ha realizado estudio de impacto ambiental? & $\mathrm{X}$ & \\
\hline ¿Se ha elaborado proyecto de rehabilitación ambiental? & & $x$ \\
\hline $\begin{array}{c}\text { ¿Se ha realizado catastro de la flora y de la fauna en el área del } \\
\text { proyecto? }\end{array}$ & & $x$ \\
\hline ¿Se han identificado los sitios patrimoniales de la comunidad? & & $x$ \\
\hline ¿Se ha caracterizado social y económicamente la comunidad? & & $x$ \\
\hline ¿Se ha elaborado un plan para el cierre de mina? & & $x$ \\
\hline ¿Existe un plan de reinserción laboral? & & $x$ \\
\hline ¿Existe un plan de reconversión profesional? & & $x$ \\
\hline $\begin{array}{l}\text { ¿Se han identificado potencialidades naturales para determinar } \\
\text { actividades alternativas? }\end{array}$ & & $\mathrm{X}$ \\
\hline ¿Se ha creado fondo para financiar futuros proyectos productivos? & & $x$ \\
\hline ¿Existe una guía que regule las reglas de seguridad minera? & $x$ & \\
\hline$\Sigma$ & 2 & 9 \\
\hline
\end{tabular}

\subsection{Indicador de compensación (IC)}

Los indicadores de compensación se tienen en cuenta en el sentido de compensar el impacto ambiental, patrimonial y cultural causado por la actividad minera y las vías de solución para mitigar estos impactos negativos en el medio, así como en la creación de actividades alternativas que sustentarán las comunidades después de que se agoten los recursos minerales.

\section{Criterios del indicador}

- EIA- El estudio de impacto ambiental establece la identificación, pronóstico y prevención de las alteraciones producidas por las actividades extractivas, desde la investigación y explotación minera hasta el procesamiento de las sustancias a beneficiar.

- PRA - El proyecto de rehabilitación minero ambiental se asocia a la idea de que el lugar alterado sea recuperado teniendo en cuenta un proyecto previo y compatible al entorno. 
- CFF- El catastro de la flora y fauna establece la identificación, cuantificación y cualificación de la flora y la fauna existente en la zona de concesión.

- SP- Los sitios patrimoniales establecen la identificación, cuantificación y caracterización de los sitios considerados patrimonio para fines culturales e investigativos.

- CSE- La caracterización socioeconómica de la comunidad establece las principales actividades económicas de la comunidad y las condiciones sociales de la población existentes en la zona de desarrollo minero.

- CM- La elaboración de plan de cierre de mina establece el conjunto de medidas o acciones llevadas a cabo durante y/o finalizada la actividad minera, con el objetivo de evitar en un largo plazo un grado de impacto ambiental significativo.

- RL- Plan de reinserción laboral establece como meta principal la colocación del trabajador desplazado en un nuevo puesto de trabajo y todas aquellas acciones que contribuyan a una adecuada habilitación del trabajador para la obtención y mantenimiento en su nuevo puesto de trabajo.

- RP- La reconversión profesional establece el entrenamiento o capacitación en un arte laboral de obra diferente del habitual.

- IPN- La identificación de potencialidades naturales establece la identificación de las potencialidades naturales de la región para determinar actividades alternativas.

- FFP- La creación del fondo para financiar futuros proyectos establece la creación de un fondo monetario para financiar proyectos de producción en el cual la empresa se convierte en socio de la comunidad.

- SM- La seguridad minera refleja las medidas de seguridad que deben tomar los trabajadores y personal técnico de la mina durante la ejecución de los trabajos de exploración y explotación.

El índice de sostenibilidad de compensación (ISC, en porcentaje) se calcula como:

$I S C(\%)=\sum \frac{[(E I A+P R A+A F F+S P+C S E+C M+R L+R P+I P N+F F T+S M)]}{11} X 100$ 


$$
\begin{aligned}
& I S C(\%)=\sum \frac{[(1+0+0+0+0+0+0+0+0+0+1)]}{11} X 100 \\
& I S C=18,2 \%
\end{aligned}
$$

\begin{tabular}{|c|c|c|}
\hline \multirow{2}{*}{ Cuestionario } & \multicolumn{2}{|c|}{ Cumple } \\
\hline & Sí & No \\
\hline $\begin{array}{l}\text { ¿La gestión de la actividad minera en la provincia es realizada } \\
\text { adecuadamente? }\end{array}$ & & $x$ \\
\hline $\begin{array}{c}\text { ¿La comunidad participa en la toma de decisiones de la empresa y en el } \\
\text { desarrollo comunitario? }\end{array}$ & & $x$ \\
\hline $\begin{array}{c}\text { ¿Las instituciones académicas participan en la resolución de los } \\
\text { problemas de la comunidad? }\end{array}$ & & $x$ \\
\hline ¿La cantidad de empleados nacionales es favorable? & $\mathrm{x}$ & \\
\hline ¿La tasa ocupacional de los empleados nacionales es favorable? & $x$ & \\
\hline ¿Los empleados han tenido formación profesional? & & $x$ \\
\hline $\begin{array}{c}\text { ¿El porcentaje de las inversiones que se realiza para la prevención y } \\
\text { salud de los empleados es favorable? }\end{array}$ & & $x$ \\
\hline ¿Los empleados de la empresa están inscritos en la seguridad social? & $x$ & \\
\hline ¿Existen profesionales de la empresa que se dedican a la actividad I+D & & $x$ \\
\hline $\begin{array}{l}\text { ¿El salario mínimo de los empleados corresponde al salario mínimo en la } \\
\text { función pública nacional? }\end{array}$ & $x$ & \\
\hline ¿La empresa respeta el conocimiento local? & & $x$ \\
\hline$\Sigma$ & 4 & 7 \\
\hline
\end{tabular}

Tabla 2. Desarrollo

\subsection{Indicador de desarrollo (D)}

El indicador de desarrollo se utilizará para medir el grado de mejoramiento del bienestar social de los trabajadores de las empresas mineras y de la población que vive en las zonas de desarrollo minero, así como el grado de la relación existente entre el gobierno, la comunidad y las empresas, incluyendo la participación de las instituciones científicas en el desarrollo comunitario.

\section{Criterios del indicador}

- FI- La fortaleza institucional establece el fortalecimiento de las instituciones públicas, la capacitación del recurso humano, la actualización de las normativas, la alianza entre instituciones públicas 
y la comunidad de modo a proveer la base de la sustentación y de consenso para el desarrollo sostenible.

- PC- El grado de participación de la comunidad en la toma de decisiones de la empresa establece el nivel de participación de la población de las comunidades en la toma de decisiones de la empresa y en el desarrollo comunitario.

- PIA- La participación de las instituciones académicas en el desarrollo comunitario establece el nivel de participación de las instituciones académicas o científicas en la resolución de los problemas de las comunidades.

- EN- La tasa de empleados nacionales establece el número de nacionales (locales) contratados por la empresa minera.

- ON- La tasa ocupacional de los empleados nacionales establece la función que el empleado ocupa en la empresa (directivo, técnico o auxiliar).

- FP- La tasa de formación profesional establece la cantidad de empleados formados profesionalmente por la empresa.

- SS- La tasa de gastos por servicio de salud establece el porcentaje de las inversiones que se realiza para la prevención de la salud de los empleados.

- AS- La tasa de asegurados socialmente establece el porcentaje de empleados de la empresa inscritos en la seguridad social.

- I+D- La tasa de Investigación y Desarrollo en el sector establece la cantidad de profesionales dedicados a la actividad I+D.

- IS- El índice salarial establece el salario mínimo pago en la empresa comparado con el salario mínimo nacional pagado por estado.

- TA- Las tecnologías apropiadas establecen el grado de consideración de las empresas al conocimiento local.

El índice de sostenibilidad del desarrollo (ISD, en porcentaje) se calcula como:

$$
I S D(\%)=\sum \frac{[(F I+P C+P I A+E N+O N+F P+S S+A S+I D+I S+T A)]}{11} X 100
$$


$I S D(\%)=\sum \frac{[(0+0+0+1+1+0+0+1+0+1+0)]}{11} X 100$

$I S D=36,4 \%$

Por tanto, el índice de sostenibilidad global (ISG, en porcentaje) obtenido es el siguiente:

$$
\begin{aligned}
& I S G(\%)=\sum \frac{[(I S G M+I S C E+I S C+I S D)]}{\text { Totaldecriterios }} X 100 \\
& I S G(\%)=\sum \frac{[(3+9+2+4)]}{43} X 100 \\
& I S G=41,8 \%
\end{aligned}
$$

Donde:

ISGM: Índice de sostenibilidad geológico minero;

ISCE: Índice de sostenibilidad en el crecimiento económico;

ISC: Índice de sostenibilidad en la compensación;

ISD: Índice de sostenibilidad en el desarrollo.

Este valor, al ser inferior al $50 \%$, indica que la actividad extractiva no es sostenible $y$, por tanto, la empresa minera debe revisar a fondo todas las acciones que lleva a cabo en aras de convertirse en una empresa sostenible.

\subsection{Información para la toma de decisiones}

Los resultados de los indicadores demuestran una realidad que exige de una inmediata intervención de cada uno de los elementos que integran la tríada gobierno-comunidad-empresa, los cuales conforman los ejes sobre los cuales se fundamentará una estrategia para el desarrollo sostenible de la industria de los minerales de la construcción en Uige. Sobre la base de estos resultados se proponen acciones para cada uno de los actores que conforman el escenario socioeconómico de la provincia.

\section{Primera acción}

- Las empresas mineras deben precisar los niveles de explotación de los yacimientos de acuerdo con la capacidad de los sistemas socioeconómicos de Uige de sustituir en las cadenas productivas 
locales, regionales o nacionales los puestos laborales que dejarán de existir cuando se agoten los yacimientos actuales.

- De igual forma las empresas tienen que establecer mecanismos que eviten sobrepasar la capacidad de recomposición natural de los recursos renovables que utilizan en el proceso de producción. Los bajos índices de sostenibilidad de la gestión minera $(27,27 \%)$ demuestran que no se tienen en cuenta las categorías que intervienen en la dimensión ambiental de la sostenibilidad que contribuyen con este indicador.

\section{Segunda acción}

- Los altos niveles del índice de sostenibilidad de crecimiento económico (ISCE), en contraste con los bajos índices de los demás indicadores, demuestran que este crecimiento económico no contribuye al desarrollo interno de la provincia. De ahí, que se recomienda que el gobierno instrumente mecanismos legales que garanticen que, de acuerdo con los convenios firmados con las empresas transnacionales y nacionales, el crecimiento de las empresas se reinvierta hacia la comunidad.

- La planificación de este desarrollo debe tener en cuenta la opinión de la comunidad como un actor clave del desarrollo sostenible de Uige, especialmente debe considerarse la opinión de las autoridades tradicionales. Estos elementos deben formar parte de una estrategia única para el desarrollo sostenible de Uige.

\section{Tercera acción}

- Identificar todas las acciones que garanticen la estabilización de las variables que facilitan las compensaciones como la vía para garantizar la sostenibilidad de la actividad minera. El bajo índice de sostenibilidad de compensación (ISC) demuestra que una vez que se agoten los materiales de la construcción en Uige las comunidades que dependen de este recurso para su crecimiento y desarrollo entrarán en un estado de muerte social.

- El gobierno será responsable de elaborar todas las normativas que sirvan de base para la elaboración de una estrategia que a través de compensaciones garantice el desarrollo sostenible de la industria de materiales de la construcción de Uige. Para ello utilizará los resultados que este indicador aporta. 


\section{Cuarta acción}

- Los bajos índices aportados por el índice de sostenibilidad de desarrollo (ISD) sugieren la necesidad de elaborar una estrategia que defina cómo los aportes al crecimiento económico de la provincia tributarán al desarrollo sostenible. Especialmente se precisa legislar la forma en que las empresas transnacionales que se asientan en la provincia implementarán mecanismos para transferir hacia la comunidad las tecnologías que utilizan y cómo el gobierno ejecutará políticas para asimilar el conocimiento que portan estas tecnologías.

- Definir en la estrategia de desarrollo sostenible que se elabore para la industria extractiva de materiales de la construcción de Uige las acciones de crecimiento económico y cómo contribuirán al desarrollo de la industria y de la comunidad. Para la determinación de estas acciones se tendrá en cuenta las diferencias existentes entre crecimiento económico y desarrollo.

\section{CONCLUSIONES}

- El sistema de indicadores propuesto permite medir el grado de desarrollo sostenible en el sector minero, teniendo en cuenta el estado técnico y organizativo de las canteras y las dimensiones ambiental, económica y social. Los indicadores de sostenibilidad obtenidos permiten medir la sostenibilidad de las empresas mineras de materiales para la construcción.

- El resultado del test de sostenibilidad aplicado en una de las empresas demuestra que la actividad extractiva se encuentra en el campo de la no sostenibilidad.

\section{REFERENCIAS}

Arango, M. y Olaya, Y. 2012: Problemas de los pasivos ambientales mineros en Colombia. Gestión y Ambiente, 15(3): 125-133.

Araña, W. 2015: La responsabilidad socio-ambiental de las empresas mineras cubanas. Ciencia \& Futuro, 5(3): 63-75.

Carmona-García, U.; Cardona-Trujillo, H. y Restrepo-Tarquino, I. 2017: Gestión ambiental, sostenibilidad y competitividad minera. Contextualización de la situación y retos de un enfoque a través del análisis del ciclo de vida. Dyna, 84(201): 50-58.

Carvajal, D. y González, A. 2003: La contribución del patrimonio geológico y minero al desarrollo sostenible. En: Villas-Boas, R. C.; González Martínez, A. y Albuquerque, G. A. (ed.). Patrimonio 
geológico y minero en el contexto del cierre de minas. Río de Janeiro: CNPq/CYTED, p. 27-50.

Herbert, J. 2006: Introducción a los fundamentos de la tecnología minera. España: Universidad Politécnica de Madrid. 131 p.

La Rotta, A. M. y Torres, M. H. 2017: Explotación minera y sus impactos ambientales en la salud. El caso de Potosí en Bogotá. Saúde em Debate, 41(112): 77-91.

Millán, E. 2013: Procedimiento para la rehabilitación mineroambiental de los yacimientos polimetálicos. Tesis doctoral. Instituto Superior Minero Metalúrgico de Moa.

Montero-Matos, J.; Restrepo-Baena, O. J. y Otaño-Nogel, J. 2017: Cierre sostenible de canteras de materiales para la construcción en Cuba. Minería y Geología, 33(4): 441-455.

Montero-Peña, J. M. 2009: El desarrollo compensado: una alternativa para el desarrollo sustentable en la minería. En: Conferencia internacional sobre minería sostenible. La Coruña: Cámara oficial Mineira de Galicia, p. 89-100.

Montes De Oca, A. y Ulloa, M. 2013: Recuperación de áreas dañadas por la minería en la cantera Los Guaos, Santiago de Cuba, Cuba. Luna Azul, (37): 74-78.

Parravicini, D. 2015: Apuntes sobre el cierre de minas. Panorama minero: enfoques, (428): 48-52. Consultado: 14/02/2018. Disponible en: https://www.palermo.edu/Archivos content/2015/derecho/2015di ciembre/parravicini_4/parravicini_cierre_de_minas.pdf

Reynaldo-Argüelles, C. L. 2012: Metodología para el diseño de indicadores económicos-ambientales en la minería del níquel. Minería y Geología, 28(2): 13-29.

Saade, M. 2013: Desarrollo minero y conflictos socioambientales. Los casos de Colombia, México y el Perú. Santiago de Chile: Naciones Unidas. Serie Macroeconomía del Desarrollo No 137. Consultado: 14/02/2018. Disponible en: https://repositorio.cepal.org/bitstream/handle/11362/5369/LCL37 06 es.pdf.

Sturla, G. y Muñoz, C. 2014: La política de agua en Chile y la gran minería del cobre. Revista Análisis Público, 4: 63-99. Consultado: 14/02/2018. Disponible en: https://mpra.ub.unimuenchen.de/id/eprint/76089. 
Urán, A. 2013: La legalización de la minería a pequeña escala en Colombia. Revista Latinoamericana de Estudios Socioambientales, (14): 255-283. Consultado: 12/03/2018. Disponible en: https://doi.org/10.17141/letrasverdes.14.2013.1004.

Wong, A. y Tost, M. 2013: Iniciativa para el desarrollo de la minería responsable. Ginebra, Suiza: Foro Económico Mundial. Consultado: 24/05/2018. Disponible en: http://www3. weforum.org/docs/WEF MM RMDI Report 2013 SP. pdf.

Zúñiga, C.; Benavides, C. y Arnáez, E. 2015: Campos Universitario como agentes de la educación para la sostenibilidad ambiental. Biocenosis, 29(1-2): 24-28. Consultado: 18/05/2018. Disponible en:

https://investiga.uned.ac.cr/revistas/index.php/biocenosis/article/ viewFile/887/775.

Recibido: 09/11/2018

Aceptado: 11/03/2019 